\title{
Phase-1 Clinical Trial Results of High-Specific-Activity Carrier-Free ${ }^{123}$ I-Iobenguane
}

\author{
Bennett B. Chin ${ }^{1}$, James F. Kronauge ${ }^{2}$, Frank J. Femia ${ }^{2}$, Jianqing Chen $^{2}$, Kevin P. Maresca², Shawn Hillier ${ }^{2}$, Neil A. Petry ${ }^{1}$, \\ Olga G. James ${ }^{1}$, Jorge D. Oldan ${ }^{1}$, Thomas Armor ${ }^{2}$, James B. Stubbs ${ }^{3}$, Michael G. Stabin ${ }^{4}$, and John W. Babich ${ }^{2}$ \\ ${ }^{1}$ Department of Radiology, Duke University Medical Center, Durham, North Carolina; ${ }^{2}$ Molecular Insight Pharmaceuticals, \\ Cambridge, Massachusetts; ${ }^{3}$ Radiation Dosimetry Systems, Alpharetta, Georgia; and ${ }^{4}$ Department of Radiology and Radiological \\ Sciences, Vanderbilt University, Nashville, Tennessee
}

\begin{abstract}
A first-in-human phase 1 clinical study was performed on 12 healthy adults with a high-specific-activity carrier-free formulation of ${ }^{123}$ iobenguane. Clinical data are presented on the behavior of this receptor-targeting imaging agent. Methods: Whole-body and thoracic planar and SPECT imaging were performed over $48 \mathrm{~h}$ for calculation of tissue radiation dosimetry and for evaluation of clinical safety and efficacy. Results: A reference clinical imaging database acquired over time for healthy men and women injected with highspecific-activity ${ }^{123}$-iobenguane showed organ distribution and whole-body retention similar to those of conventional ${ }^{123}$ |-iobenguane. The heart-to-mediastinum ratios for the high-specific-activity formulation were statistically higher than for conventional formulations, and the predicted radiation dosimetry estimations for some organs varied significantly from those based on animal distributions. Conclusion: Human normal-organ kinetics, radiation dosimetry, clinical safety, and imaging efficacy provide compelling evidence for the use of high-specific-activity ${ }^{123}$-iobenguane.
\end{abstract}

Key Words: iobenguane ${ }^{123}$; MIBG; human dosimetry; high specific activity; human distribution

J Nucl Med 2014; 55:765-771

DOI: 10.2967/jnumed.113.124057

I

obenguane, or metaiodobenzylguanidine (MIBG), is a guanethidine derivative functionally resembling norepinephrine. The drug guanethidine was developed in 1960 and was once a mainstay for the treatment of hypertension (1). It is no longer used in the United States; however, it is still licensed in some countries for the rapid control of blood pressure in a hypertensive emergency and as an intravenous nerve-blocking agent to treat chronic regional pain. Iobenguane and guanethidine are substrates for the norepinephrine transporter (NET) and accumulate by the uptake 1 mechanism into presynaptic nerve endings. Unlike norepinephrine, these drugs are protonated under physiologic conditions; therefore, they do not cross the blood-brain barrier and in vivo uptake is limited primarily to systemic neuronal tissue $(2,3)$. In

Received Apr. 11, 2013; revision accepted Nov. 5, 2013.

For correspondence or reprints contact: Bennett B. Chin, Division of Nuclear Medicine, Department of Radiology, Duke University Medical Center, Box 3808, Durham, NC 27710.

E-mail: chin0004@dm.duke.edu

Published online Mar. 13, 2014.

COPYRIGHT (c) 2014 by the Society of Nuclear Medicine and Molecular Imaging, Inc. this paradigm, with chronic administration excess iobenguane can inhibit the neuroendocrine response in hypertensive patients, but with intravenous administration it can cause a hypertensive response often identified as iobenguane-associated adverse events.

In healthy adults, the tissue density of systemic NET receptors is highest in the presynaptic neurons innervating myocytes (4). The accumulation of iobenguane in myocardial tissue is also dictated by the high fraction of aortic blood flow that enters the coronary arteries. This physiology constitutes an ideal molecular targeting mechanism for diagnosis of various cardiac diseases, including heart failure, heart transplant rejection, ischemic heart disease, dysautonomia, and drug-induced cardiotoxicity, as well as cardiac neuropathy related to diabetes mellitus and Parkinson disease (5-7). If a substantial fraction of the receptors are occupied by nonradioactive substrates, the overall uptake of radioactive tracer may be reduced. Consequently, the specific activity of ${ }^{123}$ I-iobenguane used to visualize NET receptors in the heart is a critical aspect of the formulation and efficacy of this agent.

${ }^{131}$ I-iobenguane was initially approved for the diagnostic imaging of highly NET-expressing cancers such as neuroendocrine tumors. More recently, imaging is being performed with the commercially available AdreView (GE Healthcare) using ${ }^{123}$ I. The 13.2-h physical half-life and $158-\mathrm{keV} \gamma$-photon emission provides higher-resolution images (8). However, this high-specific-activity (HSA) ${ }^{123}$ I is still bound to iobenguane in the conventional radioactive isotope exchange reacted, starting with the stable-halogencontaining precursor. The terms carrier or carrier-added refer to the fact that a significant quantity of nonradioactive (or cold) iobenguane is present in the final ${ }^{123}$ I-iobenguane formulation. In conventional isotope exchange reactions, the molar ratio of nonradioactive iobenguane to radioactive ${ }^{123}$ I-iobenguane is greater than 2,000:1. Commercial formulations contain $0.4 \mathrm{mg}$ of iobenguane sulfate per dose, which can create significant competition for the NET receptors and cause significant adverse reactions.

Hunter and Zhu $(9,10)$ used solid-state precursors to produce very HSA radiolabeled agents. The solid-phase process enables preparation of a carrier-free formulation and avoids potential competitive or toxic effects of the pharmacologically active carrier, iobenguane. Molecular Insight Pharmaceuticals is developing this carrier-free manufacturing process for iobenguane under the trade name of UltraTrace. In a preclinical meeting with the Food and Drug Administration, it was advised that the distribution and safety of the HSA formulation should be studied to demonstrate potential differences from material with a lower specific activity. A phase 1 clinical trial was performed on healthy adults to evaluate the uptake and retention in the heart at an approximately 100 -fold higher 
specific activity than that of conventional ${ }^{123}$ I-iobenguane. The secondary objectives were to determine the whole-body distribution, pharmacokinetics, safety, and excretion pathways, as well as to generate a reference patient-image database for this HSA formulation.

\section{MATERIALS AND METHODS}

\section{HSA 123/-lobenguane}

The radioactive final drug product for injection was produced under current good manufacturing practices at Molecular Insight Pharmaceuticals and shipped as a sterile, ready-to-use solution to Duke University Medical Center. No-carrier-added sodium iodide ( $\left.{ }^{123} \mathrm{I}\right)$, produced by ${ }^{124} \mathrm{Xe}(\mathrm{p}, 2 \mathrm{n}){ }^{123} \mathrm{Cs} \rightarrow{ }^{123} \mathrm{Xe} \rightarrow{ }^{123}$ I nuclear reaction, was obtained from Nordion and reacted with the HSA ${ }^{123}$ I-iobenguane solid-state precursor in acetic acid and hydrogen peroxide for $20 \mathrm{~min}$ at $85^{\circ} \mathrm{C}$ as shown in Figure 1. The reaction was filtered and purified by cation-exchange chromatography to remove the acid and peroxide and formulated in phosphate-buffered ascorbic acid solution as a stabilizer to radiolysis. Each batch was tested by high-performance liquid chromatography for radiochemical identity, purity, potency, osmolality (by freezing point depression), $\mathrm{pH}$, and excipients. The solution apyrogenicity was verified by Limulus amebocyte lysate testing using the Endosafe-PTS instrument and cartridge system (Charles River) based on the kinetic chromogenic method. Sterility was ensured by parametric release using a filter integrity test validated and confirmed in each case with incubation for 2 wk in tryptic soy broth and fluid thioglycollate medium after the drug was administered.

\section{Human Subject Eligibility and Study Design}

The Duke University Institutional Review Board approved this study, and all subjects gave written informed consent. First-in-human studies were performed with this HSA ${ }^{123}$ I-iobenguane formulation under a physician-sponsored investigational new drug application (no. 106,205). The study was approved and monitored by the Duke University Medical Center Health System Institutional Review Board under the regulations of the Health and Human Services Office for Human Research Protections (Federal Wide Assurance no. 00009025). Thirty healthy volunteers at least 21 y old were screened to obtain 12 healthy subjects ( 6 male, 6 female). Screening included medical history, physical examination, vital signs, electrocardiogram, and laboratory tests (serum creatinine, serum glutamic oxaloacetic transaminase, serum glutamate pyruvate transaminase, and total bilirubin within reference ranges). Exclusion criteria were women who were pregnant or nursing; a history of allergy to shellfish, radiographic contrast medium, or iodine; abnormal test results; enrollment in another clinical trial; receipt of a radioisotope within $30 \mathrm{~d}$; a preexisting medical condition, or medications that would reduce the likelihood of obtaining reliable data or completing the study. Medications that inhibit the uptake of iobenguane within $6 \mathrm{wk}$ of enrollment include tricyclic antidepressants, sympathomimetics, antihypertensive and cardiovascular agents, opioids, and antipsychotics. Of the 30 subjects screened, 3 withdrew, 9 had abnormal laboratory test results, 3 had abnormal electrocardiogram readings, 2 had underlying medical conditions, and 1 was enrolled in a concurrent study. A serum

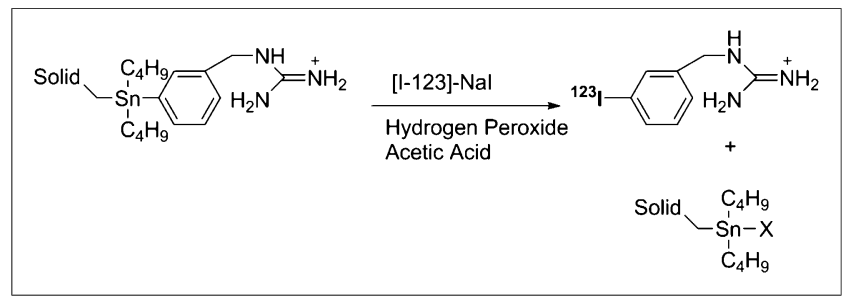

FIGURE 1. Synthetic pathway used in production of high-purity ${ }^{123 \mid-}$ iobenguane. pregnancy test for all women was performed before administration of drug. The age ranges were 26-34 y for women and 24-52 y for men.

Electrocardiograms. A 12-lead electrocardiogram and vital signs were obtained within $2 \mathrm{~h}$ before and at $1,10,15$, and $20 \mathrm{~min}$ after injection, and immediately before the 1-, 2-, 6-, and 24-h scans. Rhythm; PR, QT, QTc, and QRS intervals; and ST segment were assessed at each time point.

Laboratory Evaluations. Baseline blood and urine samples were collected $0-48 \mathrm{~h}$ before injection for comparison to 24-h postinjection and follow-up tests. Vital signs, 12-lead electrocardiograms, and blood and urine sampling were obtained before and immediately after intravenous injection, and the subjects were monitored for adverse events. No fasting or dietary restrictions before or after administration were imposed. The subjects were instructed to remain well hydrated to facilitate urine excretion. As required by the Institutional Review Board, all subjects underwent oral thyroid blocking with either 2 drops of a saturated solution of potassium iodide or 100 $\mathrm{mg}$ of iodine, 3 times daily starting $2 \mathrm{~d}$ before and continuing for $2 \mathrm{~d}$ after injection.

\section{Dosimetry}

Blood Clearance. Blood clearance parameters were determined by counting blood samples obtained at $0.25,1,4,24$, and $48 \mathrm{~h}$ after injection using WinNonlin, version 5.3 (Pharsight). Blood and plasma time-activity concentration data were analyzed by a noncompartmental method, and the peak plasma concentration of the time-concentration curve (i.e., area under the curve) and mean residence time were derived. Total organ clearance was calculated as dose divided by area under the curve, and steady-state volume of distribution was calculated as mean residence time multiplied by total organ clearance. For determination of $\mathrm{t}^{1} / 2 \alpha$ and $\mathrm{t}^{1} / 2 \beta$, a 2 -compartment model was used.

Imaging and Data Analysis. After intravenous injection of approximately $370 \mathrm{MBq}$ of HSA ${ }^{123}$ I-iobenguane, each subject underwent serial imaging at about 15-35 min and 1, 4, 24, and $48 \mathrm{~h}$ after injection. Anterior and posterior whole-body planar projections were acquired using a dual-head $\gamma$ camera (Infinia; GE Healthcare) equipped with a low-energy high-resolution collimator. Scan velocity was set to $8 \mathrm{~cm} / \mathrm{min}$ for all time points. The image matrix was $256 \times 1,024$ pixels, with a symmetric energy window of $20 \%$ centered on the $159-\mathrm{keV}$ photopeak. Each subject was imaged supine next to an imaging phantom (approximately $37 \mathrm{MBq}$ of ${ }^{123} \mathrm{I}$ in a $100-\mathrm{mL}$ saline bag) to monitor for radioactive decay, changes in camera speed, and sensitivity.

Regions of interest (ROI) for each visualized organ and the whole body were manually drawn on the first anterior whole-body image using a nuclear medicine workstation (Hermes Medical Solutions). An electronic template of these ROIs was applied to each image to reduce possible subjectivity in the ROIs between time points. A mirror image of the anterior ROIs was applied to the posterior whole-body scans.

Mean ROI counts from the imaging standard, whole body, brain, gut, heart, kidney (left kidney values were doubled to simulate both kidneys), liver, lungs, muscle, salivary glands, skeleton, spleen, thyroid, and urinary bladder contents were exported in comma-separated-value format to Radiation Dosimetry Systems for calculation of absorbed dose estimates. Changes in camera speed and decay correction were applied using an image standard.

The geometric mean (GM) of the raw count data from the anterior and posterior projections for each ROI was computed (from each pair of anterior and posterior images in the acquisition sequence). The GM of the whole-body ROI of the initial image represented $100 \%$ of the administered activity, and the percentage of the injected activity (\% IA) in each organ was calculated by dividing the GM of each organ ROI by that of the whole-body ROI. The \%IA in the image phantom of the initial whole-body image was computed analogously. For the subsequent whole-body images, no decay or camera speed corrections 
were applied directly. Instead, the GMs for each organ ROI and whole-body ROI were computed and then were divided by the GM of the whole-body ROI from the initial image (termed the precorrected \%IA). Changes in GM counts of the image phantom were solely due to radioactive decay and camera speed changes. Thus, a ratio of the GM of the phantom ROI on subsequent images to that on the initial image provided a direct correction (phantom-correction factor) for decay and camera speed changes. Therefore, multiplication of the precorrected \%IA from any subsequent image by the phantom-correction factor of that image yields organ \%IA corrected for decay and camera speed changes. Attenuation, scatter, and background subtraction were not applied.

Source Organ Time-Activity Curve Fitting. The \%IA in each source organ was mathematically simulated using biexponential functions, iteratively fit to each source organ time-activity curve using a nonlinear least-squares regression algorithm (SAAM II, version 1.2) (11). The form of the biexponential equation, $A(t)$, is as follows:

$$
A(t)=a_{1} e^{-\lambda_{1} t}+a_{2} e^{-\lambda_{2} t}
$$

where $a_{\mathrm{i}}$ is the $\mathrm{i}^{\text {th }}$ fractional uptake and $\lambda_{\mathrm{i}}$ is its associated biologic removal rate $\left(\mathrm{h}^{-1}\right)$. The time-activity integrals for the organs were obtained by integrating the curve-fit equation, $A(t)$, from $t=0$ to infinity after multiplying each term by the physical decay term (that is, $\left.e^{-\lambda_{P} t}\right)$. The effective removal rate is the sum of the biologic removal rate and the physical decay rate for the nuclide.

Total-Body Clearance and Organ Residence Times. Urinary clearance was estimated from the whole-body image data. The totalbody time-activity curve was fit to a biexponential function, and the total-body time-activity integral was calculated by integrating the equation after subtracting the estimated fecal excretion fraction (maximum \%IA value of the gut ROI seen in the 4- or 24-h images) from the more slowly clearing component of the total-body time-activity curve. Integration of this corrected total-body retention function yielded the corrected total-body time-activity integral. The parameters (fraction and associated biologic removal half-time) of the corrected total-body time-activity curves were used as input data for the dynamic (urinary) bladder model. The number of disintegrations in the urinary bladder contents was calculated using the OLINDA/EXM dynamic bladder model (12) assuming a 4.8-h void schedule. The remainder of the body activity was calculated as the corrected value for the total-body residence time minus the values for all other organs, except the urinary bladder and intestines. The gut (gastrointestinal tract) comprised the small bowel, upper large intestine (proximal colon), and lower large intestine (distal colon).

Organ Radiation Dose Estimates. The OLINDA/EXM (11) software was used to estimate the absorbed doses using its adult male dosimetry phantom. The urinary bladder was assumed to void regularly at 4.8 -h intervals, and the gut transit times of the human adult were assumed. The dose to salivary glands was determined using $\mathrm{S}$ values for a 77-g sphere.

\section{Cardiac Imaging and Data Analysis}

Cardiac planar and SPECT imaging were performed at 1 and $4 \mathrm{~h}$ after administration. Planar images were obtained and immediately followed by SPECT at both these time points. Planar images of the anterior thorax were acquired in a $256 \times 256$ matrix for $10 \mathrm{~min}$ to calculate the heart-to-mediastinum ratios $(\mathrm{H} / \mathrm{M})$. For the planar mediastinum region, a $7 \times 7$ square $\mathrm{ROI}$ was placed in the superior mediastinum with careful avoidance of the thyroid and lung. For the heart region, a polygonal ROI was manually drawn around the left ventricle including the left ventricular cavity. The planar H/M ratios were calculated from their respective mean counts per pixel in each region.

The SPECT data were acquired for approximately $20 \mathrm{~min}$ (step and shoot with $3^{\circ}$ increment per projection and $30 \mathrm{~s}$ per projection; $180^{\circ}$ acquisition) in a $128 \times 128$ matrix and reconstructed with filtered backprojection. For analysis, the mediastinum volume of interest was determined from a $7 \times 7 \times 7$ voxel volume in the superior mediastinum, again avoiding thyroid and heart. The heart volume of interest was determined from oval ROIs drawn in the transaxial plane, including the left ventricle in every plane in which it could be visualized. Total counts and counts per pixel were then calculated, and the SPECT H/M ratios were calculated from the measured counts per pixel.

\section{Cardiac Imaging Comparison with Conventional Low-Specific-Activity (LSA) ${ }^{123}$-lobenguane}

All 12 healthy volunteers also underwent a comparison cardiac imaging study with commercially available LSA ${ }^{123}$ I-iobenguane (370 $\mathrm{MBq}$, or $10.0 \mathrm{mCi}$ ) (AdreView). The LSA ${ }^{123}$ I-iobenguane study was performed at least $1 \mathrm{wk}$ after the HSA ${ }^{123}$ I-iobenguane Ultratrace study, but the two studies took place no longer than 1 mo apart. Planar and SPECT cardiac imaging acquisition and data analysis methods were the same as those reported for the HSA ${ }^{123}$ I-iobenguane.

\section{RESULTS}

\section{HSA 123|-lobenguane Test Article}

The HSA ${ }^{123}$ I-iobenguane had at least $94 \%$ radiochemical purity and no more than $5 \%$ free iodide at radioactive concentrations of $83-102 \mathrm{MBq} / \mathrm{mL}(2.25-2.75 \mathrm{mCi} / \mathrm{mL})$ at the time of calibration. The final product solution was verified as sterile and contained 3\% ascorbic acid and had a $\mathrm{pH}$ of 4.5-5.5. The osmolality was 1-1.5 times normal saline, and the level of endotoxins was less than 5.05 endotoxin units $/ \mathrm{mL}$. The most prevalent nonradioactive degradation products for iobenguane are deiodinated benzylguanidine and hydroxyl-substituted meta-hydroxybenzylguanidine. These two impurities have the same binding affinity for the NET receptor as iobenguane (13-15). The concentrations of HSA iobenguane-related species are shown in Table 1. For comparison, a conventional dose of MIBG contains all active pharmaceutical ingredient species, which yield a specific activity of $300 \mathrm{MBq} / \mu \mathrm{mol}$ (370 MBq/dose and $1.2 \mu \mathrm{mol} / \mathrm{dose}$ ), or greater than 100 times more than that of HSA ${ }^{123}$ I-iobenguane $(36,275 \mathrm{MBq} / \mu \mathrm{mol})$.

The mean and SD of the administered mass of HSA ${ }^{123}$ I-iobenguane was $1.56 \pm 0.10 \mu \mathrm{g}(0.0082-0.0122 \mu \mathrm{mol})$ of iobenguanerelated products. The mean administered Ultratrace dose was $355 \pm 20.9 \mathrm{MBq}(9.6 \pm 0.56 \mathrm{mCi})$. There were no adverse or clinically detectable pharmacologic effects in any of the 12 subjects. No significant changes in vital signs or in the results of laboratory studies or electrocardiograms were observed.

\section{Whole-Body Imaging Results and Analysis}

High-quality whole-body images were obtained up to $25 \mathrm{~h}$ after administration; 48-h images showed relatively lower counts due to physical decay and biologic excretion. The organ distributions were the same for men and women throughout $24 \mathrm{~h}$, as can be appreciated in representative male and female images in Figures 2 and 3 , respectively.

Activity cleared rapidly from the blood and exhibited a biexponential decrease in concentration, as summarized in Table 2. After reaching a peak plasma concentration at $15 \mathrm{~min}$ after injection, blood concentrations of ${ }^{123}$ I declined with a half-life of $78.4 \pm 27.5 \mathrm{~h}$ for all subjects, with no difference observed between male and female subjects; the residence time in blood was $111 \pm 39.7 \mathrm{~h}$. Mean blood clearance was $58.1 \pm 50.2 \mathrm{~mL} / \mathrm{h} / \mathrm{kg}$, indicating that HSA ${ }^{123}$ I-iobenguane was rapidly cleared. For all subjects, mean blood exposure or area under the curve from zero to infinity was $0.11 \pm$ $0.06 \mathrm{~h} \times \mathrm{MBq} / \mathrm{kg}$. The mean steady-state volume of distribution was 
TABLE 1

Summarized High-Performance Liquid Chromatography/Mass Spectrometry Concentrations of lobenguane and Related Chemical Species for HSA ${ }^{123}$ I-lobenguane $(n=6)$

\begin{tabular}{|c|c|c|c|c|c|c|}
\hline Parameter & 123I-MIBG & ${ }^{127}$ I-MIBG & All MIBG* & $\mathrm{mHBG}^{\dagger}$ & $\mathrm{BG}^{\ddagger}$ & All API species \\
\hline Content (ng/mL) & $4.7 \pm 3.1^{\S}$ & $9.6 \pm 9.1^{\S}$ & 14.3 & $13.2 \pm 3.0^{\S}$ & $362.8 \pm 55.1^{\S}$ & 390.3 \\
\hline Content (nmol/mL) & 0.0173 & 0.0348 & 0.052 & 0.0795 & 2.4187 & 2.55 \\
\hline \multicolumn{7}{|c|}{ Specific activity dose } \\
\hline $\mathrm{GBq} / \mu \mathrm{mol}$ & $5,353.3$ & $2,659.4$ & $1,775.8$ & $1,163.2$ & 38.2 & 36.26 \\
\hline $\mathrm{mCi} / \mu \mathrm{mol}$ & 144,684 & 71,876 & 47,994 & 31,439 & 1,033 & $980.4 \|$ \\
\hline \multicolumn{7}{|c|}{ 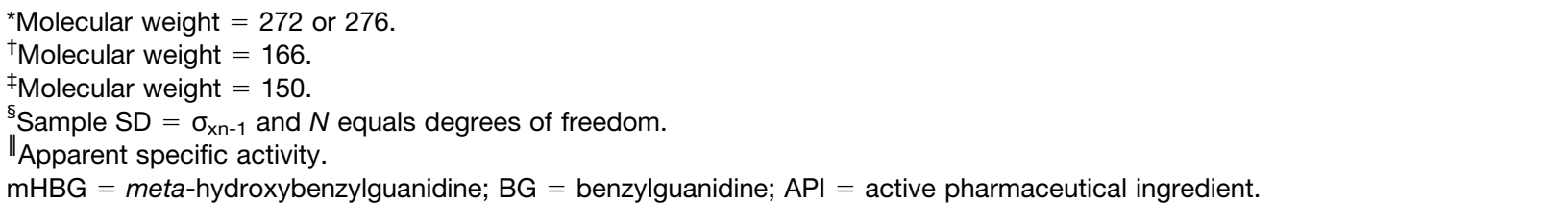 } \\
\hline
\end{tabular}

$4,853 \pm 1,180 \mathrm{~mL}$, indicating that HSA ${ }^{123}$ I-iobenguane was distributed in total-body tissue. A statistically significant difference in blood pharmacokinetics between men and women was observed for area under the curve, clearance rate, steady-state volume of distribution, total blood $\mathrm{t}^{1 / 2}$, and the $\mathrm{t}^{1 / 2} \beta$ clearance rate, with 2 -tailed $P$ values of $0.0044,0.0474,0.0169,0.0081$, and 0.0268 , respectively, mostly because of the longer $\beta$ portion of the clearance curves. A difference of about $1 \%$ in men and $2 \%$ in women for residual activity in the blood at 24-48 $\mathrm{h}$ would account for these different pharmacokinetic parameters but would have no effect on organ distribution, radiation dose calculations, or safety profile. The activity excreted in the urine (\% injected dose) ranged from 41 to 73 at $24 \mathrm{~h}$ and from 48 to 86 at $48 \mathrm{~h}$, with no significant difference observed between men and women.

\section{Planar and SPECT Cardiac Analysis}

For planar imaging including both men and women, HSA MIBG compared with LSA MIBG provided slightly higher H/M

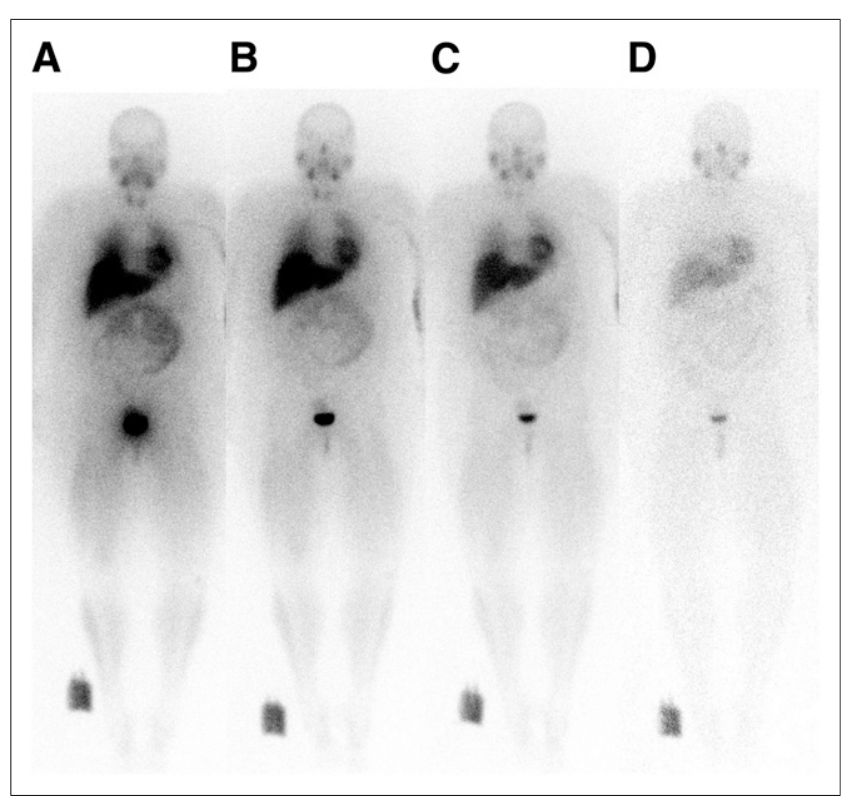

FIGURE 2. Anterior whole-body images in healthy man at $0.25 \mathrm{~h}(\mathrm{~A})$, $1 \mathrm{~h}(\mathrm{~B}), 4 \mathrm{~h}(\mathrm{C})$, and $24 \mathrm{~h}(\mathrm{D})$ after injection of HSA ${ }^{123}$-iobenguane (48-h postinjection images are not shown; calibration standard is located at right foot). (target-to-background) ratios on initial 1-h imaging ( $2.1 \pm 0.2 \mathrm{vs.}$ $2.0 \pm 0.2 ; P=0.01)$ and at $4 \mathrm{~h}(2.2 \pm 0.3$ vs. $2.0 \pm 0.2 ; P<$ 0.001). A separate, independent, repeated masked analysis of the planar images confirmed this slightly higher $\mathrm{H} / \mathrm{M}$ ratio for HSA MIBG than for LSA MIBG on both the initial 1-h imaging (2.0 \pm 0.3 vs. $1.9 \pm 0.3 ; P=0.01)$ and at $4 \mathrm{~h}(2.2 \pm 0.4$ vs. $2.0 \pm 0.3$; $P=0.01)$. Separate analyses of total counts in the ROIs for the heart and the mediastinum did not reveal significant differences between HSA MIBG and LSA MIBG. Although the mean counts per pixel in the mediastinum (background) on initial and delayed imaging were lower for HSA MIBG than for LSA MIBG, these values were not statistically significant $(P=0.08$ and 0.22 , respectively). Similarly, the calculated mean counts per pixel in the heart on initial and delayed imaging were higher for HSA MIBG than for LSA MIBG, but these differences were again not statistically significant $(P=0.67$ and 0.16 , respectively).

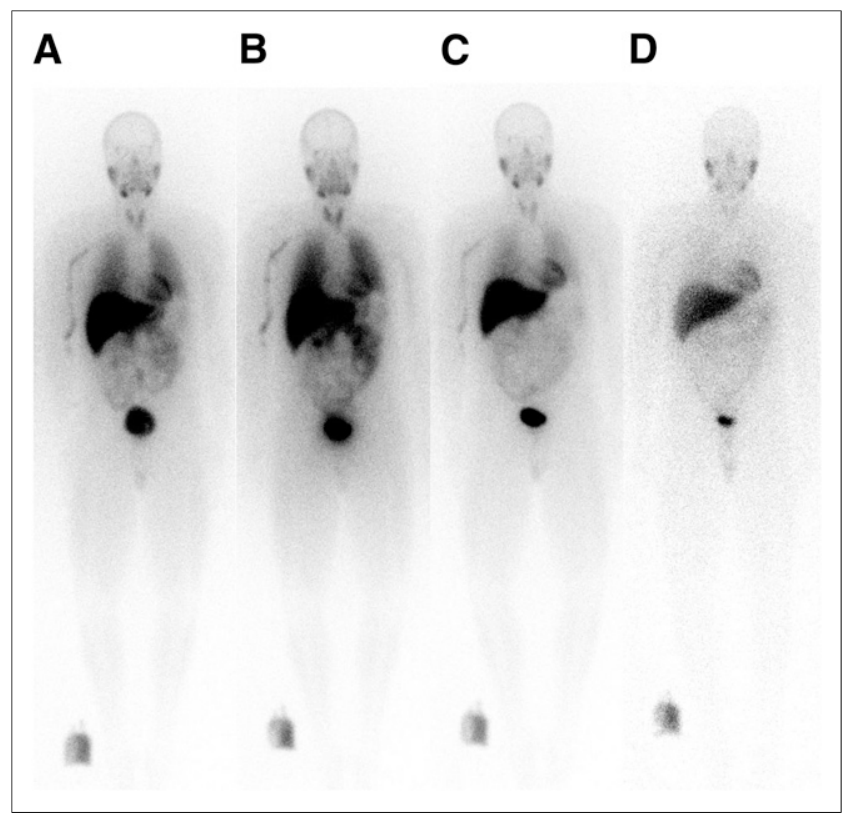

FIGURE 3. Anterior whole-body images in healthy woman at $0.25 \mathrm{~h}$ $(\mathrm{A}), 1 \mathrm{~h}(\mathrm{~B}), 4 \mathrm{~h}(\mathrm{C})$, and $24 \mathrm{~h}(\mathrm{D})$ after injection of HSA ${ }^{123}$-iobenguane (48-h postinjection images are not shown; calibration standard is located at right foot). 
TABLE 2

HSA ${ }^{123}$--lobenguane Pharmacokinetic Parameters in Healthy Adult Humans

\begin{tabular}{|c|c|c|c|}
\hline Parameter & Women $(n=6)$ & Men $(n=6)$ & All subjects \\
\hline $\operatorname{Tmax}(\mathrm{h})$ & $0.20 \pm 0.11$ & $0.25 \pm 0.12$ & $0.22 \pm 0.12$ \\
\hline Peak plasma concentration $(\mathrm{Bq} / \mathrm{mL})$ & $3,034 \pm 1,554$ & $2,553 \pm 1,628$ & $2,886 \pm 1,665$ \\
\hline Area under curve, $0-\infty(\mathrm{h} \times \mathrm{MBq} / \mathrm{mL})$ & $0.15 \pm 0.04$ & $0.08 \pm 0.04$ & $0.11 \pm 0.06$ \\
\hline Clearance $(\mathrm{mL} / \mathrm{h} / \mathrm{kg})$ & $30.3 \pm 7.16$ & $82.8 \pm 58.4$ & $58.1 \pm 50.2$ \\
\hline Steady-state volume of distribution $(\mathrm{mL} / \mathrm{kg})$ & $3,993 \pm 923.6$ & $5,550 \pm 810.2$ & $4,853 \pm 1,180$ \\
\hline$t 1 / 2(h)$ & $93.9 \pm 15.8$ & $61.4 \pm 25.5$ & $78.4 \pm 27.5$ \\
\hline Mean residence time, $0-\infty(h)$ & $133.4 \pm 22.52$ & $85.94 \pm 36.31$ & $110.7 \pm 39.7$ \\
\hline$t 1 \frac{1}{2} a(h)$ & $0.21 \pm 0.09^{*}$ & $0.24 \pm 0.10$ & $0.22 \pm 0.09$ \\
\hline$t 1 / 2 \beta(h)$ & $101.7 \pm 32.1^{*}$ & $59.6 \pm 20.6$ & $78.7 \pm 33.3$ \\
\hline Urine (\% injected dose at $24 \mathrm{~h}$ ) & $51.8 \pm 8.79$ & $55.7 \pm 10.5$ & $53.7 \pm 9.46$ \\
\hline Urine (\% injected dose at $48 \mathrm{~h}$ ) & $65.5 \pm 12.3$ & $69.1 \pm 11.8$ & $67.3 \pm 11.6$ \\
\hline
\end{tabular}

Analysis of SPECT images showed no significant differences in $\mathrm{H} / \mathrm{M}$ ratios between HSA MIBG and LSA MIBG on either 1 -h SPECT $(4.3 \pm 0.6$ vs. $4.0 \pm 0.8 ; P=0.10)$ or 4 -h SPECT $(5.5 \pm 1.0$ vs. $5.1 \pm 1.0 ; P=0.18)$. SPECT imaging of the heart showed high, homogeneous distribution throughout the myocardium for both HSA MIBG and LSA MIBG. Representative HSA MIBG 1- and 4-h delayed images are shown in Figure 4.

\section{Organ Radiation Dose Estimates and Clinical Safety Evaluations}

The organ and whole-body radiation dose estimates for the adults from HSA MIBG are summarized in Table 3. Absorbed dose calculations indicate that the critical target organs in decreasing order were the urinary bladder wall $(0.073 \mathrm{mSv} / \mathrm{MBq})$, intestinal walls $(0.031-0.071 \mathrm{mSv} / \mathrm{MBq})$, salivary glands $(0.064$ $\mathrm{mSv} / \mathrm{MBq})$, thyroid $(0.055 \mathrm{mSv} / \mathrm{MBq})$, and kidneys $(0.028 \mathrm{mSv} /$ $\mathrm{MBq}$ ), with an effective dose of $0.027 \mathrm{mSv} / \mathrm{MBq}$.

No serious adverse events occurred over the duration of the study. All potential adverse events observed either were previously documented side effects of iobenguane or were deemed unrelated to the study drug by the principal investigator. None of the 12 subjects showed any adverse or clinically detectable pharmacologic effects attributable to the drug. No significant changes in vital signs or results of laboratory studies were attributable to the drug. One subject with elevated aspartate aminotransferase enzymes (grade 2 Common Terminology Criteria for Adverse Events) $24 \mathrm{~h}$ after injection reported taking repeated high doses of an acetaminophen-diphenhydramine combination during the course of the study. Aspartate aminotransferase levels were within the reference range within $1 \mathrm{wk}$,

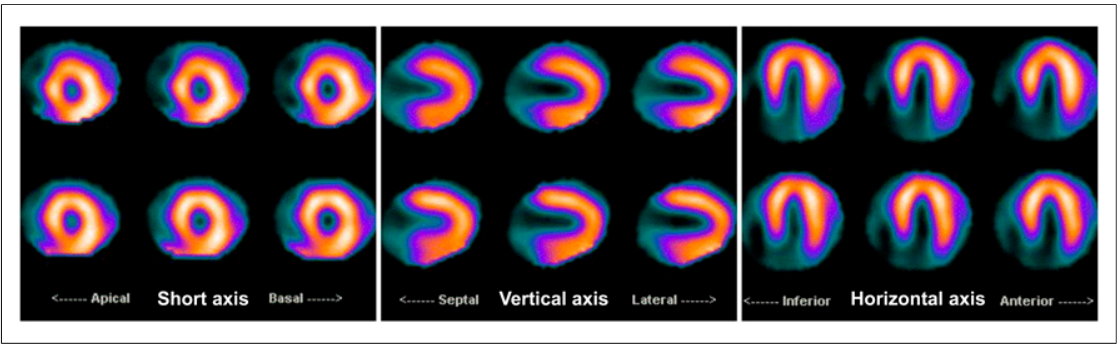

FIGURE 4. Representative mid-ventricular HSA ${ }^{123}$ /-iobenguane SPECT images in healthy $33-y-$ old woman. (Top) Early SPECT short-axis (left), vertical long-axis (middle), and horizontal longaxis (right) images. (Bottom) Corresponding delayed SPECT images in same orientations. and the event was deemed by the principal investigator to be unrelated to the study drug.

Potential grade 1 adverse reactions according to the Common Terminology Criteria for Adverse Events included mild dizziness (1 subject), low-grade fever (1 subject), rhinitis (4 subjects), elevated monocytes (1 subject), mildly elevated leukocytes (1 subject), mild hypoalbuminemia (2 subjects), hypocalcemia (1 subject), mild eosinophilia (1 subject), and developing cough (1 subject). All symptoms were mild and resolved within an additional 2 wk of follow-up, and the principal investigator deemed the events not to be related to the study drug.

\section{DISCUSSION}

Conventional formulations of LSA iobenguane that contain nonradioactive iodine-, bromine-, or chlorine-substituted benzyl guanidine result in much lower apparent specific activities, with lower target tissue uptake and potential for physiologic side effects. The HSA ${ }^{123}$ I-iobenguane process may provide the greatest benefits for ${ }^{131} \mathrm{I}$, which is made from lower-specific-activity ${ }^{131} \mathrm{I}$-sodium iodide and therapeutic agents that are administered at much higher dosages. However, the process is also well suited for the HSA isotopes, for which the labeling reactions tend to be inconsistent and give lower radiochemical yields such as ${ }^{123} \mathrm{I}$ and ${ }^{124} \mathrm{I}$. With the apparent trend to market more PET imaging equipment, the appropriately labeled NET targeting agent might be produced with the same components if the availability of ${ }^{124} \mathrm{I}$ could be achieved on a commercial scale.

Cell receptor binding experiments demonstrate a much greater uptake or target cell specificity for very HSA material. Typically, the in vivo accumulation in receptorexpressing tissue does not mimic the dramatic increase in uptake observed in cell studies in vitro because of the high first-pass extraction from the blood into the extracellular space and elimination by excretory organs. Consequently, the small fraction of the dose that is exposed initially to the target tissue overwhelmingly dictates the global organ distribution and subsequent imaging characteristics of an intravenously administered agent. Given this limitation, the highest specific activity 
TABLE 3

Descriptive Statistics for Radiation Dose Estimates (mGy/MBq) from HSA ${ }^{123}$ /-lobenguane Distribution in 12 Healthy Adults

\begin{tabular}{|c|c|c|c|c|c|}
\hline Site & Mean & Minimum & Maximum & SD & COV \\
\hline Urinary bladder wall & 7.3E-02 & $4.6 \mathrm{E}-02$ & $8.6 \mathrm{E}-02$ & $1.1 \mathrm{E}-02$ & $15 \%$ \\
\hline Lower large intestine wall & 7.1E-02 & 4.9E-02 & $9.6 \mathrm{E}-02$ & $1.2 E-02$ & $17 \%$ \\
\hline Salivary glands & $6.4 \mathrm{E}-02$ & $3.5 \mathrm{E}-02$ & $8.8 \mathrm{E}-02$ & $1.4 \mathrm{E}-02$ & $21 \%$ \\
\hline Upper large intestine wall & 6.3E-02 & 4.3E-02 & 8.4E-02 & $1.0 \mathrm{E}-02$ & $17 \%$ \\
\hline Thyroid & $5.5 \mathrm{E}-02$ & 3.9E-02 & $7.6 \mathrm{E}-02$ & $9.4 \mathrm{E}-03$ & $17 \%$ \\
\hline Small intestine & $3.1 \mathrm{E}-02$ & 2.3E-02 & $3.9 E-02$ & $4.0 \mathrm{E}-03$ & $13 \%$ \\
\hline Kidneys & $2.8 \mathrm{E}-02$ & 2.3E-02 & $4.9 E-02$ & 7.0E-03 & $25 \%$ \\
\hline Liver & 2.7E-02 & 2.2E-02 & 3.3E-02 & $3.8 \mathrm{E}-03$ & $14 \%$ \\
\hline Heart wall & 2.7E-02 & $1.9 \mathrm{E}-02$ & $3.1 \mathrm{E}-02$ & 3.3E-03 & $12 \%$ \\
\hline Osteogenic cells & $2.4 \mathrm{E}-02$ & $2.2 \mathrm{E}-02$ & $2.7 \mathrm{E}-02$ & $1.7 \mathrm{E}-03$ & $7 \%$ \\
\hline Ovaries & $2.2 \mathrm{E}-02$ & $1.8 \mathrm{E}-02$ & $2.6 \mathrm{E}-02$ & $2.0 \mathrm{E}-03$ & $9 \%$ \\
\hline Uterus & 1.9E-02 & 1.7E-02 & $2.1 \mathrm{E}-02$ & 9.7E-04 & $5 \%$ \\
\hline Gallbladder wall & 1.7E-02 & $1.6 \mathrm{E}-02$ & $1.9 \mathrm{E}-02$ & $7.0 \mathrm{E}-04$ & $4 \%$ \\
\hline Spleen & 1.6E-02 & 1.3E-02 & $2.0 \mathrm{E}-02$ & $1.9 \mathrm{E}-03$ & $12 \%$ \\
\hline Lungs & $1.5 \mathrm{E}-02$ & $1.1 \mathrm{E}-02$ & $1.8 \mathrm{E}-02$ & $1.9 \mathrm{E}-03$ & $12 \%$ \\
\hline Pancreas & $1.2 \mathrm{E}-02$ & $1.1 \mathrm{E}-02$ & 1.3E-02 & 7.4E-04 & $6 \%$ \\
\hline Adrenals & $1.2 \mathrm{E}-02$ & $1.1 \mathrm{E}-02$ & 1.3E-02 & 8.7E-04 & $7 \%$ \\
\hline Stomach wall & $1.1 \mathrm{E}-02$ & $1.0 \mathrm{E}-02$ & $1.2 \mathrm{E}-02$ & $5.6 \mathrm{E}-04$ & $5 \%$ \\
\hline Muscle & $8.8 \mathrm{E}-03$ & 8.3E-03 & $9.5 \mathrm{E}-03$ & $3.9 \mathrm{E}-04$ & $4 \%$ \\
\hline Red marrow & 8.7E-03 & 8.1E-03 & 9.3E-03 & $4.0 \mathrm{E}-04$ & $5 \%$ \\
\hline Thymus & $8.5 E-03$ & $7.4 \mathrm{E}-03$ & 9.3E-03 & $6.3 E-04$ & $7 \%$ \\
\hline Testes & 8.4E-03 & 7.9E-03 & $9.1 \mathrm{E}-03$ & $3.4 \mathrm{E}-04$ & $4 \%$ \\
\hline Breasts & $6.1 \mathrm{E}-03$ & $5.4 \mathrm{E}-03$ & $6.6 \mathrm{E}-03$ & $4.5 E-04$ & $7 \%$ \\
\hline Skin & 5.3E-03 & 4.8E-03 & $5.9 E-03$ & $3.4 \mathrm{E}-04$ & $6 \%$ \\
\hline Brain & $4.0 \mathrm{E}-03$ & 3.7E-03 & $4.6 \mathrm{E}-03$ & $3.0 \mathrm{E}-04$ & $8 \%$ \\
\hline Total body & $1.0 \mathrm{E}-02$ & $9.8 \mathrm{E}-03$ & $1.1 \mathrm{E}-02$ & $4.2 E-04$ & $4 \%$ \\
\hline Effective dose (mSv/MBq) & 2.7E-02 & 2.3E-02 & $3.0 \mathrm{E}-02$ & $1.9 \mathrm{E}-03$ & $7 \%$ \\
\hline
\end{tabular}

for a receptor-binding agent, as predicted, results in excellent imaging characteristics but does not produce a dramatic difference in nontarget tissue distribution or normal-organ radiation exposure.

Tissue radiation dosimetry estimates to normal tissue are similar for the HSA MIBG formulation and conventional LSA MIBG material, except for the intestines, for which our data yielded a 10fold higher absorbed dose than that listed in the AdreView package insert (16). The correlation with older modeling data is fairly good considering that previous radiation dose estimates were based on application of a MIRD program on animal distribution data that probably underestimate human gastrointestinal transit times $(17,18)$. The total-body exposure compared well with the literature results (16), with HSA MIBG and LSA MIBG values of 10.0 and $8.1 \mathrm{mGy} / \mathrm{MBq}$, respectively; however, the effective dose comparison of 27.0 versus $13.7 \mu \mathrm{Sv} / \mathrm{MBq}$ suggests the animal model data may underestimate the radiation dose to humans. Regardless, the radiation exposure for an imaging dose of this ${ }^{123} \mathrm{I}$-labeled agent is between 5 and 10 mrem and well below the limits for detecting measurable effects.

The planar H/M ratios for the carrier-free formulation in this study were only slightly higher than those measured for the conventional radiotracer. In a large, prospective study of patients with heart failure (AdreView Myocardial Imaging for Risk Evaluation in Heart Failure), a significant prognostic indicator of increased risk of cardiac events (heart failure progression, arrhythmic events, and cardiac death) with the conventional formulation was delayed planar H/M ratios of less than 1.6 (19). This normal cutoff value is concordant with our measurements of a normal H/M ratio on delayed planar imaging for both HSA MIBG and LSA MIBG (2.2 \pm 0.3 and $2.0 \pm 0.2$, respectively). Verberne et al. reported higher $\mathrm{H} / \mathrm{M}$ ratios in 9 healthy volunteers using a no-carrier-added formulation (20); however, these higher $\mathrm{H} / \mathrm{M}$ ratios could be attributed to acquisition on medium-energy collimators that reduce septal penetration and scatter of the higherenergy $\gamma$ photons from ${ }^{123} \mathrm{I}(21)$.

As expected, all SPECT H/M ratios were higher than their respective planar values; however, the variability in normal SPECT $\mathrm{H} / \mathrm{M}$ measurements was high, as previously reported (22). No significant difference in SPECT H/M ratios could be demonstrated when HSA MIBG and LSA MIBG were compared using this limited sample size. For planar H/M ratios, HSA MIBG showed only slightly higher $\mathrm{H} / \mathrm{M}$ ratios than the conventional formulation, but the normal limits are essentially the same as those previously determined for LSA MIBG (19). This result is important for the future development of this radiotracer in subsequent clinical trials. Finally, the good reproducibility of planar measurements is concordant with a previous report of high reproducibility (22).

\section{CONCLUSION}

The HSA ${ }^{123}$ I-iobenguane manufacturing process enables the rapid and consistent production of very HSA ${ }^{123}$ I-iobenguane at high yields, with less than $1 \%$ of the iobenguane-related substances compared with conventional formulations. The high myocardial uptake, high retention, and rapid background organ clearance demonstrate the clinical utility and safety of high-contrast SPECT 
imaging. This HSA formulation has the same tissue exposure, clinical safety, and radiation dosimetry as conventional ${ }^{123}$ I-iobenguane.

\section{DISCLOSURE}

The costs of publication of this article were defrayed in part by the payment of page charges. Therefore, and solely to indicate this fact, this article is hereby marked "advertisement" in accordance with 18 USC section 1734. The UltraTrace manufacturing process is based on proprietary precursor materials licensed exclusively by Molecular Insight from the University of Western Ontario. Research funding was provided by the National Cancer Institute of the Department of Health and Human Services under Small Business Innovation Award R44 CA 130394 and by Molecular Insight (now a subsidiary of Progenics Pharmaceuticals, Inc.). The NIH ClinicalTrials.gov Identifier is NCT01730417. No other potential conflict of interest relevant to this article was reported

\section{REFERENCES}

1. Mull RP, inventor. Ciha Pharmaceutical Products, Inc., assignee. Alkyleneiminolower alkyl-guanidines. U.S. patent 2,928,829, March 15, 1960.

2. Stehouwer JS, Goodman M. Fluorine-18 radiolabeled PET tracers for imaging monoamine transporters: dopamine, serotonin, and norepinephrine. PET Clin. 2009; 4:101-128.

3. Zeng F, Mun J, Jarkas N, et al. Synthesis, radiosynthesis, and biological evaluation of carbon-11 and fluorine-18 labeled reboxetine analogues: potential positron emission tomography radioligands for in vivo imaging of the norepinephrine transporter. J Med Chem. 2009;52:62-73.

4. Sakata K, Iida K, Mochizuki N, Ito M, Nakaya Y. Physiological changes in human cardiac sympathetic innervation and activity assessed by ${ }^{123}$ I-metaiodobenzylguanidine (MIGB) imaging. Circ J. 2009;73:310-315.

5. Joyce PI, Rizzi D, Caló G, Rowbotham DJ, Lambert DG. The effect of guanethidine and local anesthetics on the electrically stimulated mouse vas deferens. Anesth Analg. 2002;95:1339-1343.

6. Nagamachi S, Jinnouchi S, Kurose T, et al. Serial change in ${ }^{123}$ I-MIBG myocardial scintigraphy in non-insulin-dependent diabetes mellitus. Ann Nucl Med. 2002;16:33-38.

7. Stevens MJ. New imaging techniques for cardiovascular autonomic neuropathy: a window on the heart. Diabetes Technol Ther. 2001;3:9-22.

8. Kline RC, Swanson DP, Wieland DM, et al. Myocardial imaging in man with iodine-123 meta-iodobenzylguanidine. J Nucl Med. 1981;22:129-132.

9. Hunter DH, Zhu X. Polymer-supported radiopharmaceuticals: $\left[{ }^{131} \mathrm{I}\right] \mathrm{MIBG}$ and [123I]MIBG. J Labelled Comp Radiopharm. 1999;42:653-661.
10. Hunter DH, Zhu X, inventors. University of Western Ontario, assignee. Preparation of radiolabelled haloaromatics via polymer-bound intermediates. International patent CA2345710 C. December 30, 2008.

11. Stabin MG, Sparks RB, Crowe E. OLINDA/EXM: the second-generation personal computer software for internal dose assessment in nuclear medicine. $\mathrm{J} \mathrm{Nucl}$ Med. 2005;46:1023-1027.

12. Cloutier RJ, Smith SA, Watson EE, et al. Dose to the fetus from radionuclides in the bladder. Health Phys. 1973;25:147-161.

13. Barrett JA, Joyal JL, Hillier SM, et al. Comparison of high-specific-activity Ultratrace ${ }^{123} /{ }^{131} \mathrm{I}-\mathrm{MIBG}$ and carrier-added ${ }^{123} /{ }^{131} \mathrm{I}$-MIBG on efficacy, pharmacokinetics, and tissue distribution. Cancer Biother Radiopharm. 2010;25:299308.

14. Lee BC, Paik J-Y, Chi DY, Lee K-H, Choe YS. Potential and practical adrenomedullary PET radiopharmaceuticals as an alternative to meta-iodobenzylguanidine: $\mathrm{m}-\left(\omega-\left[{ }^{18} \mathrm{~F}\right]\right.$ fluoroalkyl $)$ benzylguanidines. Bioconjug Chem. 2004;15:104-111.

15. Van den Berg JD, Smets LA, Rutgers M, et al. Chemical characterization and comparative cellular effects of meta-iodobenzyl guanidine and benzyl guanidine. Cancer Chemother Pharmacol. 1997;40:131-137.

16. Package insert for AdreView iobenguane I 123 injection (GE Healthcare) 2008. OLINDA/EXM calculations based on biodistribution data from Swanson et al. Ann ICRP. 1987;18:329-331.

17. Swanson DP, Carey JE, Brown LE, et al. Human absorbed dose calculations for iodine-131 and iodine-123 labeled meta-iodobenzyl-guanidine (mIBG): a potential myocardial and adrenal medulla imaging agent. In: Proceedings of the Third International Radiopharmaceutical Dosimetry Symposium. Rockville, MD: U.S. Department of Health and Human Services, Public Health Service, Food and Drug Administration, Bureau of Radiological Health; 1981:213-224. DHHS Publication no. (FDA) 81-8166.

18. Swanson DP, Brown LE, Kline RC, Wieland DM, Carey JE, Thrall JH. The myocardial localization of radiolabeled meta-iodobenzylguanidine: an interspecies comparison. In: Proceedings of the Third International Radiopharmaceutical Dosimetry Symposium. Rockville, MD: U.S. Department of Health and Human Services, Public Health Service, Food and Drug Administration, Bureau of Radiological Health; 1981:204-212. DHHS Publication no. (FDA) 81-8166.

19. Jacobson AF, Senior R, Cerqueira MD, et al. Myocardial iodine-123 metaiodobenzylguanidine imaging and cardiac events in heart failure: results of the prospective ADMIRE-HF (AdreView Myocardial Imaging for Risk Evaluation in Heart Failure) study. J Am Coll Cardiol. 2010;55:2212-2221.

20. Verberne HJ, Sokole EB, van Moerkerken AF, et al. Clinical performance and radiation dosimetry of no-carrier-added vs carrier-added ${ }^{123}$ I-metaiodobenzylguanidine (MIBG) for the assessment of cardiac sympathetic nerve activity. Eur J Nucl Med Mol Imaging. 2008;35:798-807.

21. Inoue $\mathrm{Y}$, Abe $\mathrm{Y}$, Itoh $\mathrm{Y}$, et al. Acquisition protocols and correction methods for estimation of the heart-to-mediastinum ratio in ${ }^{123}$ I-metaiodobenzylguanidine cardiac sympathetic imaging. J Nucl Med. 2013;54:707-713.

22. Veltman CE, Boogers MJ, Meinardi JE, et al. Reproducibility of planar ${ }^{123} \mathrm{I}-$ meta-iodobenzylguanidine (MIBG) myocardial scintigraphy in patients with heart failure. Eur J Nucl Med Mol Imaging. 2012;39:1599-1608. 